\title{
COHOMOLOGY EQUATIONS AND COMMUTATORS OF GERMS OF CONTACT DIFFEOMORPHISMS
}

\author{
AUGUSTIN BANYAGA, RAFAEL DE LA LLAVE AND C. EUGENE WAYNE
}

\begin{abstract}
We study the group of germs of contact diffeomorphisms at a fixed point. We prove that the abelianization of this group is isomorphic to the multiplicative group of real positive numbers. The principal ingredient in this proof is a version of the Sternberg linearization theorem in which the conjugating diffeomorphism preserves the contact structure.
\end{abstract}

\section{INTRODUCTION}

In this section, we fix some notations, state the main result, and outline its proof.

The group $G_{n}$ of germs at $0 \in \mathbf{R}^{n}$ of $C^{\infty}$ diffeomorphisms of $\mathbf{R}^{n}$, fixing 0 , has been studied extensively: let us mention among others the well-known papers by Sternberg [St, St2], or Takens [T]. The problems considered there were to find normal forms for these germs. The theory of foliations has motivated further studies of the algebraic properties of groups of germs. (See for instance Sergeraert [S].) Indeed, an important notion in the theory of foliations is the notion of holonomy of a leaf $F$ of a codimension $n$ foliation $\mathscr{F}$ at $x_{0} \in F$ : this is a group homomorphism $H: \pi_{1}\left(F, x_{0}\right) \rightarrow G_{n}$, which, in a sense, describes the behaviour of the foliation near $F$, via various stability theorems [L]. If one considers foliations with an additional transverse structure, such as a transverse volume form, a transverse symplectic form or a transverse contact structure, one has to consider the group of germs at $0 \in \mathbf{R}^{n}$ of $C^{\infty}$ diffeomorphisms of $\mathbf{R}^{n}$, fixing 0 , and preserving the corresponding structure.

The purpose of this paper is to prove an algebraic property of a group of germs at $0 \in \mathbf{R}^{2 n+1}$ of $C^{\infty}$ diffeomorphisms of $\mathbf{R}^{2 n+1}$, fixing 0 , and preserving the natural contact structure $\omega=x_{1} d y_{1}+x_{2} d y_{2}+\cdots+x_{n} d y_{n}+d z$ of $\mathbf{R}^{2 n+1}$. As it is well known, this is no restriction because the Darboux theorem guarantees that in the neighborhood of a point we can always find coordinates in which a contact form has the canonical expression considered here.

Let $\operatorname{Diff}_{+}^{\infty}\left(\mathbf{R}^{2 n+1}, \omega, 0\right)$ be the group of all $C^{\infty}$ diffeomorphism $h: \mathbf{R}^{2 n+1} \rightarrow$ $\mathbf{R}^{2 n+1}$ such that $h(0)=0$ and $h^{*} \omega=\lambda \omega$, for some positive function $\lambda$. Let

Received by the editors November 30, 1987.

1980 Mathematics Subject Classification (1985 Revision). Primary 53C15, 58F18.

The second author was supported in part by NSF grant No. DMS 85-04984.

The third author was supported in part by NSF grant No. DMS 86-02001. 
$\operatorname{Diff}_{+}^{\infty}\left(\mathbf{R}^{2 n+1}, \omega, 0\right)_{*}$ be the subgroup of $\operatorname{Diff}_{+}^{\infty}\left(\mathbf{R}^{2 n+1}, \omega, 0\right)$ formed by those elements $h$ which are isotopic to the identity through $\operatorname{Diff}_{+}^{\infty}\left(\mathbf{R}^{2 n+1}, \omega, 0\right)$. (Probably, these two groups are the same: this question will be treated by one of us elsewhere.)

Throughout this paper, we will study the group $G$ of germs at $0 \in \mathbf{R}^{2 n+1}$ of elements of $\operatorname{Diff}_{+}^{\infty}\left(\mathbf{R}^{2 n+1}, \omega, 0\right)_{*}$.

Let $G_{1}$ be the normal subgroup of $G$ formed by those germs which are tangent to the identity at 0 , and $Q=G / G_{1}$ the quotient group.

Following Lychagin [Ly], we identify the group $Q$ with an extension by $\mathbf{R}^{2 n}$ of the conformal symplectic group $\operatorname{CSp}(n, \mathbf{R})$. It follows that the abelianization $H_{1}(Q)=Q /[Q, Q]$ is isomorphic to the multiplicative group of positive real numbers $\mathbf{R}^{+}$.

Let $\left[G, G_{1}\right]$ be the group generated by commutators $[g, h]=g h g^{-1} h^{-1}$, with $g \in G, h \in G_{1}$. Most of this paper is devoted to proving the following

Main Lemma (weak version). $G_{1}=\left[G, G_{1}\right]$.

In fact, we will prove a stronger result. For any real number $\lambda>0$, we define the contact homothety with factor $\lambda$ to be the diffeomorphism $\varphi_{\lambda}: \mathbf{R}^{2 n+1} \rightarrow$ $\mathbf{R}^{2 n+1}: \varphi_{\lambda}(\underline{x}, \underline{y}, z)=(\sqrt{\lambda} \underline{x}, \sqrt{\lambda} \underline{y}, \lambda z)$ where $\underline{x}=\left(x_{1}, \ldots, x_{n}\right), \underline{y}=\left(y_{1}, \ldots\right.$, $\left.y_{n}\right)$. Clearly, $\varphi_{\lambda}^{*} \omega=\lambda \omega$ and $\varphi_{\lambda} \in \operatorname{Diff}_{+}^{\infty}\left(\mathbf{R}^{2 n+1}, \omega, 0\right)_{*}$. We denote again by $\varphi_{\lambda}$ the germ at 0 of $\varphi_{\lambda}$. We will prove the following

Main Lemma (strong version). Given $h \in G_{1}$, sufficiently close to the germ of the identity and any $\lambda>1$, there is an $u \in G_{1}$ such that $h=\left[\varphi_{\lambda}^{-1}, u\right]=$ $\varphi_{\lambda}^{-1} \cdot u \cdot \varphi_{\lambda} \cdot u^{-1}$.

Observe that, writing $f=h \varphi_{\lambda}$, the lemma says that $f=u \varphi_{\lambda} u^{-1}$, i.e. $f$ is conjugate to its linear part (remember that $h$ is tangent to the identity at the origin), in some neighborhood of the origin, so that really our Main Lemma is a linearization theorem.

The best known of such linearization theorems are due to Sternberg [St, St2]. These results do not apply to our situation, because the nonresonance relations required by those theorems are violated here. The reason that our result is true has to depend on the fact that since $h$ is also a contact diffeomorphism, many of the obstructions to regularity in Sternberg's theorem do not arise. Thus any proof must use heavily the fact that $h$ is contact. (Having said that, we believe the result is still somewhat unexpected since $\varphi_{\lambda}$ has more relations among its eigenvalues than are necessary for the preservation of the contact structure.)

One other feature in which our linearization theorem differs from the versions of Sternberg's theorem of which we are aware is that if the diffeomorphism we are given is in $C^{k}$ (and $k$ is sufficiently large) we can conclude that the linearization is $C^{k-1}$. It seems that the standard methods ("scattering") would only allow us to conclude that the conjugating diffeomorphism is $C^{\alpha k}$ with 
$\alpha$ a constant less than one determined by the spectrum of the linearization [Ne, p. 53]. By other methods, it seems to be possible to prove $\alpha=1 / 2$ for contractions [Se]. We plan to come back to these issues in a future paper [BLW] written specifically from the point of view of linearization theorems.

The way we exploit the contact structure is very similar to the way the symplectic structure was used in [LMM]: using the canonical contact form, we translate the conjugacy problem between diffeomorphisms into a cohomology equation among functions of the type

$$
\Gamma_{t}-\lambda_{t}\left(\Gamma_{t} \circ f_{t}\right)=\Phi_{t}, \quad t \in[0,1]
$$

to be solved in $\Gamma_{t}$ and where $f_{t}$ is a given isotopy of contact diffeomorphisms $f_{0}=\varphi_{\lambda} ; f_{1}=h \varphi_{\lambda}$ and $\lambda_{t}, \Phi_{t}$ are functions manufactured out of $f_{t}$ and, hence also given.

The solution of this equation constitutes the heart of this paper, and will be given in $\S 5$. The Main Lemma, the fact that $H_{1}(Q) \simeq \mathbf{R}^{+}$and standard results on group extensions [HS], yield our main result (see the end of §2):

Theorem 1. The abelianization $H_{1}(G)$ of the group of germs at $0 \in \mathbf{R}^{2 n+1}$ of elements of $\operatorname{Diff}_{+}^{\infty}\left(\mathbf{R}^{2 n+1}, \omega, 0\right)_{*}$, is isomorphic to the multiplicative group $\mathbf{R}^{+}$.

Using the techniques of [BP and $\mathrm{P}]$ we obtain the following corollary of our main theorem.

Theorem 2. The abelianization $H_{1}\left(\operatorname{Diff}_{+}^{\infty}\left(\mathbf{R}^{2 n+1}, \omega, 0\right)_{*}\right)$ of

$$
\operatorname{Diff}_{+}^{\infty}\left(\mathbf{R}^{2 n+1}, \omega, 0\right)_{*}
$$

is isomorphic to the multiplication group $\mathbf{R}^{+}$.

The proof of Theorem 2 is presented in Appendix A.

One of the interests of a theorem like this one lies in the fact that the homology of various diffeomorphism groups plays an important role in the study of the Haefliger classifying space via the Mather-Thurston Theory [MA]. Note that the homology of groups of diffeomorphisms with compact support plays an even more important role. Unfortunately the calculation of the abelianization of the group of contact diffeomorphisms with compact support remains an open problem.

\section{SOME OBVIOUS HOMOMORPHISMS}

Let $h \in \operatorname{Diff}_{+}^{\infty}\left(\mathbf{R}^{2 n+1}, \omega, 0\right)$. Then $h^{*} \omega=\lambda \omega$ for some positive function $\lambda$. The correspondence $h \mapsto \lambda(0)$ is a surjective group homomorphism

$$
a: \operatorname{Diff}_{+}^{\infty}\left(\mathbf{R}^{2 n+1}, \omega, 0\right) \rightarrow \mathbf{R}^{+} .
$$

Indeed if $h_{1}, h_{2} \in \operatorname{Diff}_{+}^{\infty}\left(\mathbf{R}^{2 n+1}, \omega, 0\right), h_{i}^{*} \omega=\lambda_{i} \omega, i=1,2$, then

$$
\left(h_{1} h_{2}\right)^{*} \omega=h_{2}^{*}\left(h_{1}^{*} \omega\right)=h_{2}^{*}\left(\lambda_{1} \omega\right)=\left(\lambda_{1} \circ h_{2}\right) h_{2}^{*} \omega=\left(\lambda_{1} \circ h_{2}\right) \cdot \lambda_{2} \omega \text {. }
$$


Therefore

$$
a\left(h_{1} h_{2}\right)=\left(\left(\lambda_{1} \circ h_{2}\right) \cdot \lambda_{2}\right)(0)=\lambda_{1}(0) \cdot \lambda_{2}(0) .
$$

Let $\lambda \in \mathbf{R}^{+}$, if $\varphi_{\lambda}: \mathbf{R}^{2 n+1} \rightarrow \mathbf{R}^{2 n+1}$ is the contact homothety $(\underline{x}, \underline{y}, z) \mapsto$ $(\sqrt{\lambda} \underline{x}, \sqrt{\lambda} \underline{y}, \lambda z)$, then $a\left(\varphi_{\lambda}\right)=\lambda$. Hence $a$ is a surjective homomorphism as claimed.

The next obvious homomorphism $D_{0}: \operatorname{Diff}_{+}^{\infty}\left(\mathbf{R}^{2 n+1}, \omega, 0\right) \rightarrow \operatorname{GL}(2 n+1, \mathbf{R})$ is given by taking the differential at $0 \in \mathbf{R}^{2 n+1}$ of members of

$$
\operatorname{Diff}_{+}^{\infty}\left(\mathbf{R}^{2 n+1}, \omega, 0\right) \text {. }
$$

$D_{0}$ is a homomorphism by the chain rule since the origin is fixed. Let $Q=$ $D_{0}\left(\operatorname{Diff}_{+}^{\infty}\left(\mathbf{R}^{2 n+1}, \omega, 0\right)\right)$ be the image of the homomorphism $D_{0}$.

A description of the group $Q$ can be found for instance in [Ly]. It goes as follows: Let $(M, \omega)$ be a $(2 n+1)$-dimensional manifold equipped with a contact form $\omega$. There exists a unique vector field $E$ on $M$, called the Reeb field of $\omega$, such that $i(E) \omega=1$ and $i(E) d \omega=0$; here $i(\cdot)$ denotes the interior product. The quotient bundle $H(M)=T(M) / E$ is called the horizontal bundle. The tangent space $T_{x} M$ at $x \in M$ splits into a direct sum: $E_{x} \oplus H_{x}(M)$. A $C^{\infty}$ diffeomorphism $h: M \rightarrow M$ is a contact diffeomorphism if and only if it preserves $H(M)$, i.e. $T \times h$, the tangent map of $h$ at $x$ will map $H_{x}(M)$ into $H_{h(x)} M$. Let $x_{0} \in M$ be a fixed point of $h$ and let $A_{h}$ be the restriction of $T_{x_{0}} h$ to $H_{x_{0}}$. Let $\Omega_{x_{0}}$ be the restriction of $d \omega$ to $H_{x_{0}}$. Then $\Omega_{x_{0}}$ is a linear symplectic form (on the $2 n$-dimensional vector space $H_{x_{0}}$ ). Since $h^{*} \omega=\lambda \omega$ we have

$$
h^{*}(d \omega)=d\left(h^{*} \omega\right)=d \lambda \wedge \omega+\lambda d \omega .
$$

Restricting everything to $H_{x_{0}}$, we get

$$
A_{h}^{*} \Omega_{x_{0}}=\lambda\left(x_{0}\right) \Omega_{x_{0}}
$$

since $\left.\omega\right|_{H_{x_{0}}}=0$. Therefore, the restriction $A_{h}$ of $T_{x_{0}} h$ to $H_{x_{0}}$ is a conformal symplectic transformation, with factor $\lambda\left(x_{0}\right)$. Let $v=w+b E_{x_{0}}$ be the image of $T_{x_{0}} h$ of $E_{x_{0}}$ where $w$ is horizontal and $b$ is a coefficient

$$
\begin{aligned}
\left(h^{*} \omega\right)\left(x_{0}\right)\left(E_{x_{0}}\right) & =\omega\left(h\left(x_{0}\right)\right)\left(T_{x_{0}} h\right)\left(E_{x_{0}}\right) \\
& =\omega\left(x_{0}\right)\left(w+b E_{x_{0}}\right)=b .
\end{aligned}
$$

On the other hand

$$
\left(h^{*} \omega\right)\left(x_{0}\right)\left(E_{x_{0}}\right)=(\lambda \omega)\left(x_{0}\right)\left(E_{x_{0}}\right)=\lambda\left(x_{0}\right) .
$$

Therefore $D_{x_{0}} h$ has the following form:

$$
\left(\begin{array}{c|c}
A_{h} & w \\
\hline 0 & \lambda\left(x_{0}\right)
\end{array}\right)
$$


where $A_{\lambda}^{*} \Omega_{x_{0}}=\lambda\left(x_{0}\right) \Omega_{x_{0}}$ and $w \in \mathbf{R}^{2 n}$. Therefore $D_{x_{0}} h$ is completely determined by the couple

$$
\left(A_{h}, w\right) \in \operatorname{CSp}(n, \mathbf{R}) \times \mathbf{R}^{2 n}
$$

where $\operatorname{CSp}(n, \mathbf{R})$ is the conformal symplectic group. In fact, we have a group extension:

$$
0 \rightarrow \mathbf{R}^{2 n} \stackrel{i}{\rightarrow} Q \stackrel{P}{\rightarrow} \operatorname{CSp}(n, \mathbf{R}) \rightarrow I
$$

where $i: \mathbf{R}^{2 n} \rightarrow Q$ is given by

$$
i(v)=\left(\begin{array}{l|l}
I & v \\
\hline 0 & 1
\end{array}\right)
$$

and $P: Q \rightarrow \operatorname{CSp}(n, \mathbf{R})$ by

$$
\left(\begin{array}{l|l}
A & w \\
\hline 0 & \lambda
\end{array}\right) \mapsto A
$$

Consider the map $q: Q \rightarrow \mathbf{R}^{+}$

$$
q\left(\begin{array}{l|l}
A & w \\
\hline 0 & \lambda
\end{array}\right)=\lambda
$$

Since

$$
\left(\begin{array}{c|c}
A & a \\
\hline 0 & \lambda
\end{array}\right)\left(\begin{array}{c|c}
B & b \\
\hline 0 & \mu
\end{array}\right)=\left(\begin{array}{c|c}
A B & A b+\mu a \\
\hline 0 & \lambda \mu
\end{array}\right),
$$

$q$ is an homomorphism into the multiplicative group $\mathbf{R}^{+}$. This homomorphism is onto since for each $\lambda \in \mathbf{R}^{+}$, we have

$$
q\left(\begin{array}{c|c}
\sqrt{\lambda} I & 0 \\
\hline 0 & \lambda
\end{array}\right)=\lambda .
$$

Clearly, we have the following commutative diagrams:

$$
\operatorname{Diff}_{+}^{\infty}\left(\mathbf{R}^{2 n+1}, \omega, 0\right) \stackrel{D_{0}}{\rightarrow} Q
$$

The following fact is probably well known:

Proposition 1. The abelianization $H_{1}(Q)$ is isomorphic to the multiplicative group $\mathbf{R}^{+}$.

Proof. Since $q: Q \rightarrow \mathbf{R}^{+}$is surjective, we need to verify that $\operatorname{Ker} q=[Q, Q]$. But clearly $[Q, Q] \subset \operatorname{Ker} q$ because $\mathbf{R}^{+}$is abelian. To show that $\operatorname{Ker} q \subset$ $[Q, Q]$, we observe that an element of $\operatorname{Ker} q$ has the form

$$
\left(\begin{array}{l|l}
A & C \\
\hline 0 & 1
\end{array}\right)
$$

where $A \in \mathrm{Sp}(n, \mathbf{R})$ (the symplectic group), $C \in \mathbf{R}^{2 n}$. Clearly

$$
\left(\begin{array}{c|c}
A & C \\
\hline 0 & 1
\end{array}\right)=\left(\begin{array}{c|c}
A & 0 \\
\hline 0 & 1
\end{array}\right)\left(\begin{array}{c|c}
I & A^{-1} C \\
\hline 0 & 1
\end{array}\right) .
$$


Elements of the form $\left(\frac{A \mid 0}{0 \mid 1}\right)$ with $A \in \operatorname{Sp}(n, \mathbf{R})$ form a group isomorphic to $\operatorname{Sp}(n, \mathbf{R})$. Since $\operatorname{Sp}(n, \mathbf{R})$ is a perfect group (a classical fact [D]), $\left(\left.\frac{A}{0}\right|_{1} ^{0}\right)$ is a product of commutators of elements of the same type.

Now, given any $u \in \mathbf{R}^{2 n}$, we write

$$
\left(\begin{array}{l|l}
I & u \\
\hline 0 & 1
\end{array}\right)
$$

as a commutator. Let $A$ be a $(2 n \times 2 n)$-symplectic matrix with a real eigenvalue $k$ and pick an eigenvector $b$ corresponding to $k$, i.e. $A b=k b$. Choose $\mu \in \mathbf{R}^{+}, \mu \neq 1, k$ and define

$$
d=\left(\frac{\mu}{\mu-\sqrt{\mu}}\right)\left[u+\left(1-\frac{k}{\mu}\right) b\right] \in \mathbf{R}^{2 n} .
$$

Then a direct computation shows:

$$
\left(\begin{array}{l|l}
I & u \\
\hline 0 & 1
\end{array}\right)=\left(\begin{array}{l|l}
A & d \\
\hline 0 & 1
\end{array}\right)\left(\begin{array}{c|c}
\sqrt{\mu} I & b \\
\hline 0 & \mu
\end{array}\right)\left(\begin{array}{l|l}
A & d \\
\hline 0 & 1
\end{array}\right)^{-1}\left(\begin{array}{c|c}
\sqrt{\mu} I & b \\
\hline 0 & \mu
\end{array}\right)^{-1} .
$$

We would like to thank $\mathrm{L}$. Vaserstein for conversations on this section.

Consider the group $G$ of germs at $0 \in \mathbf{R}^{2 n+1}$ of elements

$$
h \in \operatorname{Diff}_{+}^{\infty}\left(\mathbf{R}^{2 n+1}, \omega, 0\right)_{*} .
$$

The homomorphisms above are defined on $G$ and we have a commutative diagram

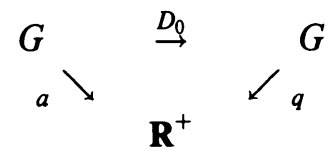

Our main result is the following:

Theorem 1. The kernel of the homomorphism $a: G \rightarrow \mathbf{R}^{+}$is equal to $[G, G]$, the commutator subgroup of $G$, i.e. the abelianization $H_{1}(G)$ of $G$ is isomorphic to the multiplicative group $\mathbf{R}^{+}$.

Proof of Theorem 1 (assuming the Main Lemma). Let $G_{1}$ be the group of those germs $h \in G$ which are tangent to the identity at $0 \in \mathbf{R}^{2 n+1}$. By definition $G_{1}=\operatorname{Ker} D_{0}$. Therefore, we obtain an extension

$$
1 \rightarrow G_{1} \rightarrow G \stackrel{D_{Q}}{\rightarrow} Q \rightarrow 1 .
$$

This yields an exact sequence

$$
\rightarrow G_{1} /\left[G_{1}, G\right] \rightarrow H_{1}(G) \rightarrow H_{1}(Q) \rightarrow 0 .
$$

See [HS]. By the Main Lemma (weak form), $G_{1} /\left[G_{1}, G\right] \simeq 0$ and by Proposition $1, H_{1}(Q) \simeq \mathbf{R}^{+}$. The exact sequence $(*)$ yields $H_{1}(G) \simeq \mathbf{R}^{+}$. 
The remainder of the paper is therefore devoted to prove the Main Lemma.

\section{SOME CONTACT GEOMETRY}

In this section we introduce some concepts that will play a dominant role later such as "contact isotopies" and review the contact geometry we will need. Some expositions of contact geometry we found useful are [G, Li, Ly2].

An isotopy of a smooth manifold $M$ is a $C^{\infty}$ map $F: M \times I \rightarrow M$ (where $I=[0,1])$ such that $\forall t \in I \quad f_{t}: M \rightarrow M: f_{t}(x)=F(x, t)$ is a $C^{\infty}$ diffeomorphism and, moreover the mapping $t \rightarrow f_{t}$ is $C^{\infty}$ when the space of diffeomorphisms is given the natural $C^{\infty}$ topology.

We say that $F$ is a compactly supported isotopy if there is a compact $K \subset M$ such that $F(x, t)=x, \forall t \in I, x \in M-K$. Let $(M, \omega)$ be a contact manifold, an isotopy of $M$ is a contact isotopy if $f_{t}$ is a contact diffeomorphism for each $t$. An isotopy $F$ will be also denoted by the corresponding family of diffeomorphisms $f_{t}$.

Any such isotopy determines a family of vector fields $\mathscr{F}_{t}$ defined by

$$
\mathscr{F}_{t}(x)=d f_{t} / d t \circ f_{t}^{-1}(x)
$$

so that $f_{t} \circ f_{0}^{-1}$ is the time $t$-map of the time dependent vector field $\mathscr{F}_{t} . f_{0}$ and $\mathscr{F}_{t}$ determine $f_{t}$ by the uniqueness theorem of ODE's. The isotopy $F$ is compactly supported if and only if $\mathscr{F}$ has compact support.

Let $(M, \omega)$ be a contact manifold, i.e. a smooth manifold $M$ equipped with a contact form $\omega$. If $f_{t}$ is a contact isotopy, there is a smooth family of positive functions $\lambda_{t}=\lambda_{t}^{\left(f_{t}\right)}$ such that $f_{t}^{*} \omega=\lambda_{t} \cdot \omega$. Let $L_{X}$ denote the Lie derivative in the direction of a vector field $X$. Since

$$
\frac{d}{d t}\left(f_{t}^{*} \omega\right)=f_{t}^{*}\left(L_{\mathscr{F}_{t}} \omega\right)
$$

$L_{\mathscr{F}_{t}} \omega=\left(f_{t}^{-1}\right)^{*}\left(\dot{\lambda}_{t} \omega\right)$, where $\dot{\lambda}_{t}=d \lambda_{t} / d t$. Because $\left(f_{t}^{-1}\right)^{*} \omega=\omega /\left(\lambda_{t} \circ f_{t}^{-1}\right)$, we see that $L_{\mathscr{T}_{1}} \omega=\gamma_{t} \omega$ with $\gamma_{t}=\left\{(d / d t)\left(\ln \lambda_{t}\right)\right\} \circ f_{t}^{-1}$.

Let us now recall some facts discussed already in the introduction.

On any contact manifold $(M, \omega)$, there exists a unique vector field $Z$, called the Reeb vector field such that

$$
i(Z) \omega=1 \text { and } i(Z) d \omega=0 .
$$

Here $i(\cdot)$ is the interior product operation. Any vector field parallel to $Z$ is called a vertical vector field. A horizontal vector field is a vector field $X$ such that $i(X) \omega=0$. A 1-form $\alpha$ on $M$ such that $i(Z) \alpha=0$ is called a semibasic form. The correspondence $\xi \stackrel{d \omega}{\mapsto} i(\xi) d \omega$ is an isomorphism between horizontal vector fields and semibasic forms [Li].

Let $\mathfrak{L}_{\omega}(M)$ denote the Lie algebra of contact vector fields, i.e. those vector fields $X$ on $M$ such that $L_{X} \omega=\lambda \omega$ for some function $\lambda$. Then $i(X) \omega \in$ $C^{\infty}(M)$ and the correspondence

$$
\Phi: \mathfrak{L}_{\omega}(M) \rightarrow C^{\infty}(M) \quad X \mapsto i(X) \omega
$$

is an isomorphism. See [Li]. 
Given $H \in C^{\infty}(M)$, it is clear that $(i(Z) d H) \omega-d H$ is a semibasic 1-form.

For $H \in C^{\infty}(M)$, the vector field $\mathscr{H} \in \mathfrak{L}_{\omega}(M)$ such that $\Phi(\mathscr{H})=\omega(\mathscr{H})=$ $H$ is given by

$$
\mathscr{H}=H Z+(d \omega)^{-1}((i(Z) d H) \omega-d H) .
$$

We will call $H$ the generator of $\mathscr{H}$. Clearly, this proves

Proposition 2. Given $H \in C^{\infty}(M)$ and if $\mathscr{H} \in \mathfrak{L}_{\omega}(M)$ is the contact vector field such that $\omega(\mathscr{H})=H$, then

$$
\|\mathscr{H}\|_{C^{r}} \leq K_{r}\|H\|_{C^{r+1}}, \quad r>1,
$$

where the constant $K_{r}$ depends only on $r$.

Given a contact isotopy $f_{t}$ with associated family of contact vector fields $\mathscr{F}_{t}$, we denote by $F_{t}$ the family of generating functions of $\mathscr{F}_{t}$, i.e. $\omega\left(\mathscr{F}_{t}\right)=F_{t}$. We also call $F_{t}$ the generator of the isotopy. Clearly, we have the following proposition.

Proposition 3. If $f_{t}$ is a compactly supported isotopy

$$
\begin{aligned}
& \frac{1}{K_{l, r}} \sup _{t \in[-1,1]}\left\|\left(\frac{\partial}{\partial t}\right)^{l} F_{t}\right\|_{C^{r}} \\
& \quad \leq \sup _{t \in[-1,1]}\left\|\left(\frac{\partial}{\partial t}\right)^{l}\left(f_{t} \circ f_{0}^{-1}\right)\right\|_{C^{r}} \leq K_{l, r} \sup _{t \in[-1,1]}\left\|\left(\frac{\partial}{\partial t}\right)^{l} F_{t}\right\|_{C^{r+1}} \\
& \text { for } l \geq 0, r \geq 1
\end{aligned}
$$

and

$$
\sup _{t \in[-1,1]}\left\|\left(\frac{\partial}{\partial t}\right)^{l} \lambda_{t}^{(f)}\right\|_{C^{r}} \leq K_{l, r} \sup _{t \in[-1,1]}\left\|\left(\frac{\partial}{\partial t}\right)^{l} F_{t}\right\|_{C^{r+1}}, \quad l \geq 1 .
$$

Proof. The first inequalities are obvious if one realizes that the way of constructing $\mathscr{F}_{t}$ out of $F_{t}$ is using derivatives and identifications and the way of constructing $F_{t}$ out of $\mathscr{F}$ is just contraction of indices.

Moreover, we have seen that if $f_{t}^{*} \omega=\lambda_{t} \omega$, for some positive family of functions $\lambda_{t}$, then

$$
L_{\mathscr{F}_{t}} \omega=\gamma_{t} \omega
$$

where $\gamma_{t}=(d / d t)\left(\ln \lambda_{t}\right) \circ f_{t}^{-1}$.

Contracting with the Reeb vector field $Z$ in both sides of $(*)$, we see that

$$
\gamma_{t}=i(Z) L_{\mathscr{F}_{t}} \omega=L_{\mathscr{F}_{t}} i(Z) \omega-i([Z, \mathscr{F}]) \omega=-i\left(\left[Z, \mathscr{F}_{t}\right]\right) \omega .
$$

Recall that $i(Z) d \omega=0$. Hence

$$
0=(i(Z) d \omega)\left(\mathscr{F}_{t}\right)=d \omega\left(Z, \mathscr{F}_{t}\right)=Z \cdot \omega\left(\mathscr{F}_{t}\right)-\mathscr{F}_{t} \cdot \omega(Z)-\omega\left(\left[Z, \mathscr{F}_{t}\right]\right) .
$$

Since $\omega(Z)=1$, and $\omega(\mathscr{F})=F_{t}$, we get

$$
\gamma_{t}=-L_{Z}\left(F_{t}\right)=-Z \cdot F_{t} \text {. }
$$


The inequalities in the propositions then follow. Notice that then the constant $K_{l, r}$ can be obtained from geometric properties of the manifold and the form $\omega$, and hence can be chosen uniformly for all the isotopies whose support is in a fixed compact set.

Proposition 4. Let $f$ be a contact diffeomorphism with $f^{*} \omega=\lambda^{(f)} \omega$, and $\mathscr{H} \in$ $\mathfrak{L}_{\omega}(M)$ a contact vector field with generating function $H$. Then $f_{*} \mathscr{H}$ is a contact vector field with generating function $\left(\lambda^{(f)} \cdot H\right) \circ f^{-1}$.

Proof. If $\varphi_{t}$ is the local 1-parameter group generated by $\mathscr{H}_{t}$ then $\varphi_{t}$ is a contact transformation and $f_{*} \mathscr{H}$ generates $f \circ \varphi_{t} \circ f^{-1}$ which is a contact transformation; therefore $f_{*} \mathscr{H}$ is a contact vector field.

In general if $\alpha$ is a 1-form and $X$ is a vector field, $f: M \rightarrow M$ a diffeomorphism, $\left(f_{*} X\right)(x)=\left(T_{f^{-1}(x)} f\right)\left(X\left(f^{-1}(x)\right)\right)$, hence

$$
\begin{aligned}
\left(i\left(f_{*} X\right) \alpha\right)(x) & =\alpha(x)\left(f_{*} X\right)(x) \\
& =\alpha(x)\left(\left(T_{f^{-1}(x)} f\right) \cdot X\left(f^{-1}(x)\right)\right. \\
& =\left(f^{*} \alpha\right)\left(X\left(f^{-1}(x)\right)\right) .
\end{aligned}
$$

Here

$$
\begin{aligned}
i\left(f_{*} \mathscr{H}\right) \omega(x) & =\left(f^{*} \omega\right)(\mathscr{H})\left(f^{-1}(x)\right) \\
& =(\lambda \omega)(\mathscr{H})\left(f^{-1}(x)\right)=\lambda\left(f^{-1}(x)\right) \cdot H\left(f^{-1}(x)\right),
\end{aligned}
$$

i.e. $i\left(f_{*} \mathscr{H}\right) \omega=(\lambda \cdot H) \circ f^{-1}$.

Remark. The same calculation holds if $f$ is replaced by a contact isotopy $f_{t}$ and $\mathscr{H}$ is replaced by a family $\mathscr{H}_{t}$ of contact vector fields with generating functions $H_{t}$. Then we have if $f_{t}^{*} \omega=\lambda_{t} \omega$

$$
i\left(\left(f_{t}\right)_{*} \mathscr{H}_{t}\right) \omega=\left(\lambda_{t} \cdot H_{t}\right) \circ f_{t}^{-1} .
$$

Proposition 5. For any two isotopies $f_{t}, g_{t}$, not necessary contact, let $\mathscr{H}_{t}, \mathscr{F}_{t}, \mathscr{G}_{t}$ be the family of vector fields defined by $h_{t}=f_{t} \cdot g_{t} ; f_{t}$, and $g_{t}$. Then $\mathscr{H}_{t}=$ $\mathscr{F}_{t}+\left(f_{t}\right)_{*} \mathscr{G}_{t}$. If $f_{t}, g_{t}$ are contact isotopies and $H_{t}, F_{t}, G_{t}$ are the generating functions of $\mathscr{H}_{t}, \mathscr{F}_{t}, \mathscr{G}_{t}$, then $H_{t}=F_{t}+\left(\lambda_{t}^{(f)} \cdot G_{t}\right) \circ f_{t}^{-1}$.

Proof. The first part of the proposition is obtained directly by differentiations and some manipulations. The second part is an immediate consequence of formula $(+)$.

By using repeatedly the previous propositions, we can derive a calculus which translates the identities among contact isotopies into identities between generating functions. We summarize this calculus in

Proposition 6. Let $f_{t}$ and $g_{t}$ be contact isotopies, $F_{t}$ and $G_{t}$ their generating functions, and $\varphi$ a contact diffeomorphism.

(a) $A_{t}=F_{t}+\left(\lambda_{t}^{(f)} G_{t}\right) \circ f_{t}^{-1}$ is the generating function of $a_{t}=f_{t} \circ g_{t}$.

(b) $B_{t}=-F \circ f_{t} / \lambda_{t}^{(f)}$ is the generating function of $b_{t}=f_{t}^{-1}$. 
(c) $C_{t}=F_{t} / \lambda^{(\varphi)} \circ \varphi$ is the generating function of $c_{t}=\varphi^{-1} \circ f_{t} \circ \varphi$.

(d) $D_{t}=\left(1 / \lambda_{t}^{(g)}\right)\left[F_{t}+\left(\lambda_{t}^{(f)} G_{t}\right)\right] \circ g_{t}$ is the generating function of $d_{t}=g_{t}^{-1} \circ$ $f_{t} \circ g_{t}$.

(e) $E_{t}=F_{t}$ is the generating function of $e_{t}=f_{t} \circ \varphi$.

Remark. The converse of these propositions is also true: if some isotopies are related at $t=0$ as in the hypothesis of the previous proposition and their generating functions satisfy the identities in the conclusion, the relation is true for all $t \in I$.

Remark. Notice also that, if the generator of an isotopy has compact support, so do the associated vector fields and vice versa.

Hence, an isotopy $f_{t}$ with $f_{0}=\mathrm{Id}$, is compactly supported if and only if the generator has compact support.

Remark. The regularity assumptions in the previous proposition can be considerably relaxed from our standing assumption that everything is $C^{\infty}$. (Such an improvement will simplify matters in $§ 5$.)

We recall from standard ordinary differential equation theory that if the family of vector fields $\mathscr{F}_{t}(x)$ is continuous in $t$ but $C^{\infty}$ in $x$, then $f_{t}(x)$ solving $(d / d t) f_{t}(x)=\mathscr{F}_{t}\left(f_{t}(x)\right) ; f_{0}(x) \in C^{\infty}$ given, is $C^{\infty}$ in $x$ and $C^{1}$ on $t$.

So that, if our purpose is to study regularity of the endpoints of an isotopy, it suffices to prove continuity with respect to the variable $t$. R].)

(A particularly elegant proof of this regularity statement for O.D.E.'s is [I,

Out of these remarks and the identification theorem, we can immediately deduce

Proposition 7 [BP]. Let $(M, \omega)$ be a contact manifold, $A \subseteq M$ a closed subset and $f_{t}$ a contact isotopy with $f_{0}=\mathrm{Id}$. Then there exists a contact isotopy $g_{t}$ with compact support which agrees with $f_{t}$ on $A$.

Proof. It suffices to give $g_{t}$ a generator which agrees with that of $f_{t}$ on $\overline{U f_{t}(A)}$ and has compact support.

\section{A COHOMOLOGY EQUATION}

Let $(M, \omega)$ be a contact manifold and $\varphi$ be a contact diffeomorphism. We perturb $\varphi$ by composing it with a contact diffeomorphism $h$ with compact support, close enough to the identity, i.e. we consider $f=h \circ \varphi$ and wish to see if our perturbed system $f$ is stable, i.e. if $f$ is conjugate to $\varphi$ within the group of contact transformations; in other words, we seek a contact diffeomorphism $g$ such that

$$
g^{-1} \circ f \circ g=\varphi
$$

If we succeed, then $h$ will be a commutator of contact diffeomorphisms since $f=g \circ \varphi \circ g^{-1} ;$ hence $h=g \circ \varphi \circ g^{-1} \circ \varphi^{-1}$. By Lychagin [Ly], there is a 
compactly supported contact isotopy $h_{t}$ between $h$ and the identity. To solve $(*)$, we seek a contact isotopy $g_{t}, g_{0}=$ identity, with compact support, such that

$$
g_{t}^{-1} \circ f_{t} \circ g_{t}=\varphi
$$

where $f_{t}=h_{t} \circ \varphi$. Clearly $\left(*^{\prime}\right)$ can be rewritten as

$$
f_{t} \circ g_{t}=g_{t} \circ \varphi \text {. }
$$

Let $F_{t}, G_{t}$, the families of generating functions for the isotopies $f_{t}, g_{t}$, and respectively. Proposition 6 yields that $(* *)$ is equivalent to

$$
F_{t}+\left(\lambda_{t}^{(f)} \cdot G_{t}\right) \circ f_{t}^{-1}=G_{t} .
$$

Hence, it suffices to find a $G_{t}$ satisfying

$$
G_{t}-\left(\lambda_{t}^{(f)} \cdot G_{t}\right) \circ f_{t}^{-1}=F_{t}
$$

which is a "cohomology" equation. Its solution will yield $G_{t}$, which, in turn, determines the contact isotopy $g_{t}\left(g_{0}=\mathrm{id}\right)$ solving $(* *)$.

An alternative strategy to solve the stability equation $(*)$ would be to use the classical "scattering" method [St, $\mathrm{Ne}]$. We construct $g$ by taking the limit

$$
g(x)=\lim _{n \rightarrow \infty}\left(f^{-n} \circ \varphi^{n}\right)(x) \text {. }
$$

The existence of this limit can be established if $f$ and $\varphi$ are tangent to a sufficiently high order at the origin. Unfortunately the existence of such a high order tangency is not apparent due to the complications introduced by the contact structure. On the other hand the method of generating functions which we use implements these constraints automatically.

To establish that $g$ preserves the contact structure one would need to modify the estimates to show that $f^{-n} \circ \varphi^{n}$ converges in $C^{1}$ sense. (This is harder than showing it converges in $C^{0}$ and that the limit is a $C^{1}$ function.) This difficulty is overcome in [C], work which we were not aware of until the present paper was completed and [BLW] was underway. Even after this second problem is cured the first remains and thus we have not attempted to use this method. Furthermore we believe that the method used in the present paper will have additional applications.

\section{Proof of the Main Lemma (strong version)}

In this section we prove the Main Lemma (strong version), that is: given $h \in G$, close enough to the germ of the identity and $\varphi_{\lambda}$ a contact homothety, of factor $\lambda>1$ we can find $u \in G_{1}$ such that $u^{-1} \circ(h \circ \varphi) \circ u=\varphi$.

Notice that such a result can be interpreted as saying that the germ $h \circ \varphi$ can be conjugated by a contact diffeomorphism to its linear part. Hence, the result is a linearization theorem. From this point of view, it is worth remarking that the fact that $\varphi$ is a homothety does not play any significant role and we could have 
worked with any $\varphi$ whose derivative at zero is expanding and whose eigenvalues are not "too different" in magnitude. Since the emphasis of this paper is not normal form theory but the algebraic structure of $G_{1}$, we do not give the precise conditions.

By the remarks in the previous section, we can study the equation $u^{-1}$ 。 $(h \circ \varphi) \circ u=\varphi$ not among germs but rather among diffeomorphisms representing those germs.

We can assume that $h$ is compactly supported and we need to find $u$ that satisfies the equation in a neighborhood of zero.

We can furthermore assume that $h_{t}$-an isotopy connecting the identity to $h$-has compact support.

If we call $f_{t}=h_{t} \circ \varphi$, it suffices (even if it is, in principle, not necessary) to find another isotopy $u_{t}$ satisfying $f_{t} \circ u_{t}=u_{t} \circ \varphi$ in a neighborhood of zero.

The advantage of attacking the problem for isotopies-which in principle is harder-is that, as was shown previously this equation is equivalent to a cohomology equation between families of functions.

The equation we want to study is

$$
U_{t}-\left(\lambda_{t}^{(f)} U_{t}\right) \circ f_{t}^{-1}=F_{t}
$$

where $F_{t}, U_{t}$ are the generating functions of the isotopy $f_{t}, u_{t}$.

Notice that, in contrast to the conjugacy equation among diffeomorphisms, this equation is linear!

Notice that, if we consider (1)-even for a fixed $t$-for an arbitrary function, it could have no solution even at the level of power series. However $F_{t}$ is the generating function of a contact isotopy tangent to the identity, and hence, has extra properties, which we will establish immediately.

The rest of the section is devoted to the solution of the cohomology equation for families that satisfy the previously established properties. We first study the cohomology equation for fixed-but arbitrary-values of the parameter $t$ and show it has smooth solutions. Then, we study dependence on parameters. By the last remark after Proposition 6, it would suffice to prove continuity. We will, nevertheless prove $C^{\infty}$ dependence.

Proposition 8. If $f_{t}$ is a contact isotopy of $\mathbf{R}^{2 n+1}$ and $f_{0}=\mathrm{id}$, then the following two statements are equivalent:

(A) $f_{t}(0)=0$ and all elements of the isotopy are tangent to the identity at zero.

(B) The generating function satisfies $F_{t}(0)=0$ and

$$
F_{t}(\underline{x}, \underline{y}, z)=a(t) z^{2}+\Phi(\underline{x}, \underline{y}, z, t),
$$

where all functions on the right-hand side of this last equation are $C^{\infty}$ and the Taylor series for $\Phi$ (at the origin) begins with cubic terms in $(\underline{x}, \underline{y}, z)$. 
Proof. We begin by showing that (A) implies (B). Let $\underline{x}=\left(x_{1}, \ldots, x_{n}\right), \underline{y}=$ $\left(y_{1}, \ldots, y_{n}\right)$ and $(\underline{x}, \underline{y}, z) \in \mathbf{R}^{2 n+1}$. Write $f_{t}$ as

$$
f_{t}(\underline{x}, \underline{y}, z)=\left(\underline{x}+\underline{l}^{x}(\underline{x}, \underline{y}, z, t), \underline{y}+\underline{l}^{y}(\underline{x}, \underline{y}, z, t), z+l^{z}(\underline{x}, \underline{y}, z, t)\right) .
$$

The fact that $f_{t}(0)=0$ and that $f_{t}$ is tangent to the identity at the origin implies immediately that $l^{\alpha}(\underline{0}, \underline{0}, 0)=0$ and

$$
\partial_{\beta} l^{\alpha}(\underline{0}, \underline{0}, 0)=0
$$

for $\alpha, \beta=x_{j}, y_{j}$ or $z$. (Here $\partial_{x_{j}}$ denotes partial differentiation with respect to $x_{j}$ and similarly for $y_{j}$ and $z$.) Since $f_{t}^{*} \omega=\lambda_{t}^{(f)} \omega$, with $\omega=\underline{x} \cdot d \underline{y}+d z$ we can derive the identities

$$
\begin{aligned}
\left\{\sum_{j=1}^{n}\left(x_{j}+l^{x_{j}}(\underline{x}, \underline{y}, z)\right) \cdot \frac{\partial l^{y_{j}}}{\partial x_{k}}(\underline{x}, \underline{y}, z)\right\}+\frac{\partial l^{z}}{\partial x_{k}}(\underline{x}, \underline{y}, z) & =0, \\
k & =1, \ldots, n,
\end{aligned}
$$

and

$$
\begin{gathered}
x_{k}\left\{\sum_{j=1}^{n}\left(x_{j}+l^{x_{j}}(\underline{x}, \underline{y}, z)\right) \cdot \frac{\partial l^{y_{j}}}{\partial z}(\underline{x}, \underline{y}, z)+\left(1+\frac{\partial l^{z}}{\partial z}(\underline{x}, \underline{y}, z)\right)\right\} \\
=\left\{\sum_{j=1}^{n}\left(x_{j}+l^{x_{j}}(\underline{x}, \underline{y}, z)\right)\left(\delta_{j, k}+\frac{\partial l^{y_{j}}}{\partial y_{k}}(\underline{x}, \underline{y}, z)\right)\right\}+\frac{\partial l^{z}}{\partial y_{k}}(\underline{x}, \underline{y}, z), \\
k=1, \ldots, n .
\end{gathered}
$$

Differentiating (5.4) with respect to $x_{l}$ we find

$$
\begin{aligned}
\frac{\partial^{2} l^{z}}{\partial x_{l} \partial x_{k}}(\underline{x}, \underline{y}, z)= & -\left\{\sum_{j=1}^{n}\left(\delta_{j, l}+\frac{\partial l^{x_{j}}}{\partial x_{l}}(\underline{x}, \underline{y}, z)\right) \cdot \frac{\partial l^{y_{j}}}{\partial x_{k}}(\underline{x}, \underline{y}, z)\right\} \\
& +\sum_{j=1}^{n}\left(\delta_{j, k}+\lambda^{y_{j}}(\underline{x}, \underline{y}, z)\right) \cdot \frac{\partial^{2} l^{y_{j}}}{\partial x_{k} \partial x_{l}}(\underline{x}, \underline{y}, z) .
\end{aligned}
$$

If we now set $\underline{x}=\underline{y}=z=0$, and use (5.3) we find $\left(\partial^{2} l^{z} / \partial x_{l} \partial x_{k}\right)(\underline{0}, \underline{0}, 0)=0$, for $k, l=1, \ldots, n$. If we differentiate (5.4) with respect to $y_{l}$ we obtain $\left(\partial^{2} l^{2} / \partial y_{l} \partial x_{k}\right)(\underline{0}, \underline{0}, 0)=0$ for $k, l=1, \ldots, n$. Finally, if we differentiate (5.4) with respect to $z$, we find $\left(\partial^{2} l^{z} / \partial x_{k} \partial z\right)(\underline{0}, \underline{0}, 0)=0$. Similarly, if we differentiate (5.5) with respect to $y_{l}, z$, we find that

$$
\frac{\partial^{2} l^{z}}{\partial y_{l} \partial y_{k}}(\underline{0}, \underline{0}, 0)=\frac{\partial^{2} l^{z}}{\partial y_{l} \partial z}(\underline{0}, \underline{0}, 0)=0, \quad l, k=1, \ldots, n .
$$

Note that these remarks, combined with (5.3) imply that the only possible quadratic terms in the Taylor series of $l^{z}$ are proportional to $z^{2}$. 
Recall now that $\mathscr{F}_{t}=d f_{t} / d t \circ f_{t}^{-1}$ and that $f_{t}$ (and hence $f_{t}^{-1}$ ) is tangent to the identity at the origin. We see that this observation, combined with (5.3) and the results above imply that if $\mathscr{F}_{t}^{\underline{x}}, \mathscr{F}_{t}^{\underline{y}}, \mathscr{F}_{t}^{z}$ are the $\underline{x}, \underline{y}$, and $z$-components of $\mathscr{F}_{t}$ then

(i) $\mathscr{F}_{t}^{\underline{x}}$ and $\mathscr{F}_{t}^{\underline{y}}$ have Taylor series at the origin that begin with quadratic terms.

(ii) $\mathscr{F}_{t}^{z}$ has a Taylor series at the origin beginning with cubic terms, except possibly for terms proportional to $z^{2}$.

Recall finally that $F_{t}=i\left(\mathscr{F}_{t}\right) \omega=\underline{x} \cdot \mathscr{F}_{t}^{\underline{y}}+\mathscr{F}_{t}^{z}$. Thus by (i) and (ii), the only quadratic terms in the Taylor series of $F_{t}$ at the origin are proportional to $z^{2}$. This proves $(\mathrm{B})$.

We now show that (B) implies (A). By the formula which expresses the contact vector field $\mathscr{F}_{t}$ in terms of $F_{t}$ we see that each component of $\mathscr{F}_{t}$ has a Taylor series at the origin beginning with quadratic terms or higher. Thus, when we integrate to recover $f_{t}$, all the $f_{t}$ 's will be tangent to the identity at the origin.

We now return to the proof of the Main Lemma. We need to find a $C^{\infty}$ solution $u_{t}$ of (5.1). Recall that the function $F_{t}$ in (5.1) is the generating function of $f_{t}=h_{t} \circ \varphi$. The isotopy $h_{t}$ satisfies the hypotheses of Proposition 8. By part (e) of Proposition 6, the generating functions of $f_{t}$ and $h_{t}$ are equal. Thus,

$$
F_{t}(\underline{x}, \underline{y}, z)=a(t) z^{2}+\text { cubic terms. }
$$

Define $\hat{\mathscr{U}}_{t}(\underline{x}, \underline{y}, z)=a(t) z^{2} /\left(1-\left(\lambda^{0}\right)^{-1}\right)$. (Here $\lambda^{0}$ is the contact factor associated with the contact diffeomorphism $\varphi$.) Note that $\hat{\mathscr{U}}_{t}$ is $C^{\infty}$ in $(\underline{x}, \underline{y}, z, t)$. Note further that

$$
F_{t}-\widehat{\mathscr{U}}_{t}+\left(\lambda_{t}^{(f)} \widehat{\mathscr{U}}_{t}\right) \circ f_{t}^{-1}
$$

has a Taylor series at the origin that begins with cubic terms since $f_{t}$ is tangent to the identity at the origin while the Taylor series of $\lambda_{t}^{(f)}$ is of the form

$$
\lambda_{t}^{(f)}=\lambda^{0}+\text { linear terms }
$$

For convenience we cut $\hat{\mathscr{U}}_{t}$ off so that it has compact support. Let $\chi_{1}(\underline{x}, \underline{y}, z)$ be a $C^{\infty}$ function that is 1 inside $B_{1}$, the ball of radius 1,0 outside $B_{2}$ and monotonically decreasing. Set $\mathscr{U}_{t}=\chi_{1} \widehat{\mathscr{U}}_{t}$. (We specify the radius of $B_{2}$ below.)

Let $u_{t}$ be the isotopy with generating function $\mathscr{U}_{t}$ and $u_{0}=$ id. Note that by Proposition $8 u_{t}$ is tangent to the identity at the origin. Now set $r_{t}=u_{t}^{-1} \circ f_{t} \circ u_{t}$. We see that $r_{0}=\varphi$ and more importantly its generating function (by Proposition 6) is

$$
R_{t}=\frac{1}{\lambda_{t}^{(u)}}\left[F_{t}-\mathscr{U}_{t}+\left(\lambda_{t}^{(f)} \mathscr{U}_{t}\right) \circ f_{t}^{-1}\right] \circ u_{t},
$$


which by (5.6) has a Taylor series at the origin that begins with cubic terms. Note that this fact, combined with the estimate on the vector field in terms of the generating function following Proposition 2, and standard estimates on the solution of differential equations in terms of the associated vector fields implies that for any $\delta>0$ we can choose $\rho>0$ such that

$$
\sup _{t \in[0,1]}\left\|r_{t}-\varphi\right\|_{B_{\rho}} \leq \delta \text { and } \sup _{t \in[0,1]}\left\|D\left(r_{t}-\varphi\right)\right\|_{B_{\rho}} \leq \delta,
$$

where $D$ represents differentiation with respect to $\underline{x}, \underline{y}, z$. We now choose $\delta$ small enough that $(1+\delta)^{26}<\lambda^{0}$ and fix $\rho$ accordingly.

To show $h_{t}$ can be written as a commutator it will suffice to find an isotopy $g_{t}$ conjugating $r_{t}$ to $\varphi$, i.e. $\varphi=g_{t}^{-1} \circ r_{t} \circ g_{t}$. The existence of $g_{t}$ will follow from the existence of a smooth generating function $G_{t}$ satisfying

$$
R_{t}-G_{t}+\left(\lambda_{t}^{(r)} G_{t}\right) \circ r_{t}^{-1}=0 .
$$

We first show how one can solve the simpler equation

$$
\widetilde{R}_{t}-\widetilde{G}_{t}+\lambda^{0} \widetilde{G}_{t} \circ r_{t}^{-1}=0,
$$

where $\widetilde{R}_{t}$ is no longer necessarily the generating function of $r_{t}$, but is still a $C^{\infty}$ function whose Taylor series at the origin begins with cubic terms. We then indicate how to reduce (5.8) to (5.9).

Note that if the Taylor series of $F_{t}$ contained terms of the form $x_{i} y_{j}, x_{i} x_{j}$, or $y_{i} y_{j}$, it would not be possible to eliminate them from the cohomology equation.

Lemma 5.1. Let $r$ be a $C^{\infty}$ mapping $\mathbf{R}^{2 n+1} \rightarrow \mathbf{R}^{2 n+1}$ leaving the origin fixed. Assume also that $r$ is close to a contact homothety $\varphi$ of factor $\lambda>1$ in the sense that $\|r-\varphi\|_{C^{1}\left(B_{\zeta}\right)}<\delta$, where $B_{\zeta}$ is a ball around the origin of radius $\zeta$ and $\delta$ is small enough so that $(1+\delta)^{26}<\lambda$.

If $R$ is a $C^{\infty}$ function, such that the Taylor expansion at the origin begins with cubic terms, then, the function

$$
G(\underline{x}, \underline{y}, z)=\lim _{N \rightarrow \infty} \sum_{k=0}^{N} \lambda^{k} R\left(r^{-k}(\underline{x}, \underline{y}, z)\right)
$$

converges uniformly in $B_{\zeta}$, is $C^{\infty}$ there and, restricted to this ball, is a solution of $R-G+\lambda G \circ r^{-1}=0$. Moreover, if $R_{t}, r_{t}$ are continuous families of functions satisfying the hypothesis of the lemma, the corresponding $G_{t}$ 's depend continuously on $t$.

Proof of Lemma 5.1. The proof consists in showing that

$$
\sum_{k=0}^{\infty}\left\|\lambda^{k} D^{m} R \circ r^{-k}\right\|_{C^{0}\left(B_{\delta}\right)}<\infty
$$

for $m \geq 0$. 
Then, by Weierstrass, $M$ test, the limit exists and is $C^{\infty}$. It is also easy to check that it satisfies the functional equation obtained in the lemma.

Defining $G^{[N]}=\sum_{k=0}^{N} \lambda^{k} R \circ r^{-k}$ we have

$$
R-G^{[N]}+\lambda G^{[N]} r^{-1}=\lambda^{(N+1)} R \circ r^{-(N+1)} .
$$

The functional equation is recovered by taking the limit $N \rightarrow \infty$.

The continuous dependence on parameters follows from the observation that the bounds we obtain for each one of the terms in the series are going to be uniform when $R_{t}, r_{t}$ move in a small neighborhood. If we consider the terms in the sum defining $G_{t}$ as functions of $t$, the series will converge uniformly.

The uniform convergence will be consequence of the following lemmas. Notice also that, a more careful reading of our lemmas would also allow us to derive bounds for $\|G\|_{C^{k}}$ in terms of $\|R\|_{C^{k}}$. This would be important for other strategies of proof. (See the remark at the end of this section.)

Lemma 5.2. Under the hypothesis of Lemma 5.1, we have

$$
\left\|D^{m} r^{-k}\right\|_{C^{0}\left(B_{\xi}\right)} \leq C_{m}(1+\delta)^{k} \lambda^{(m-k) / 2} k^{m}
$$

and the constants $C_{m}$ are independent of $k$ and can be picked uniformly for $r$ in a $C^{m}\left(B_{r}\right)$ ball.

Lemma 5.3. Under the hypothesis of Lemma 5.1

$$
\begin{aligned}
\left\|R \circ r^{-k}\right\|_{C^{0}\left(B_{\xi}\right)} & \leq K_{0}(1+\delta)^{3 k} \lambda^{-3 k / 2}, \\
\left\|D R \circ r^{-k}\right\|_{C^{0}\left(B_{\xi}\right)} & \leq K_{1}(1+\delta)^{3 k} \lambda^{-k}, \\
\left\|D^{2} R \circ r^{-k}\right\|_{C^{0}\left(B_{\xi}\right)} & \leq K_{2}(1+\delta)^{3 k} \lambda^{-k / 2}, \\
\left\|D^{m} R \circ r^{-k}\right\|_{C^{0}\left(B_{\xi}\right)} & \leq K_{m}, \quad m \geq 3,
\end{aligned}
$$

where the constants $K_{m}$ are again independent of $k$ and can be picked uniformly when $r$ ranges in a sufficiently small ball in $C^{m}\left(B_{\zeta}\right)$.

Once we have Lemma 5.2 and Lemma 5.3-whose proof we will postponewe can easily prove Lemma 5.1 .

Notice that the absolute convergence (5.10) when $m=0$ is an immediate consequence of the first part of Lemma 5.3. This guarantees $G$ is continuous.

Then, it suffices to prove it for $m$ large.

Using Faa-di Bruno's formula [AR] we have for $m \geq 1$

$$
\begin{aligned}
& \left\|\lambda^{k} D^{m}\left(R \circ r^{-k}\right)\right\|_{C^{0}\left(B_{\xi}\right)} \\
& =\lambda^{k}\left\|\sum_{\substack{q=1 \\
i_{1}+\cdots+i_{q}=m}}^{m} \sum_{\substack{i_{1} \cdots i_{q} \geq 1 \\
i_{1}+\cdots i_{q}}} \sigma_{i_{1} \ldots}\left(D^{q} R\right) r^{-k}\left(D^{i_{1}} r^{-k}\right) \cdots\left(D^{i_{q}} r^{-k}\right)\right\|_{C^{0}\left(B_{\zeta}\right)}
\end{aligned}
$$

where the $\sigma$ 's are computable numbers, whose exact value does not matter now. 
Now, we use Lemmas 5.2 and 5.3 to bound all terms in the sum in $q$. If $q \geq 3$, using the last part of Lemma 5.3 we can bound

$$
\begin{aligned}
& \left\|\sum_{\substack{i_{s} \cdots i_{q} \geq 1 \\
i_{1}+\cdots+i_{1}=m}} \sigma_{i_{1} \cdots i_{q}}\left(D^{q} R\right) \circ r^{-k}\left(D^{i_{1}} r^{-k}\right) \cdots\left(D^{i_{q}} r^{-k}\right)\right\|_{C^{0}\left(B_{\xi}\right)} \\
& \quad \leq \sum_{\substack{i_{1} \cdots i_{q} \geq 1 \\
i_{1}+\cdots+i_{q}=m}} \sigma_{i_{1} \cdots i_{q}} K_{q} C_{i_{1}} \cdots C_{i_{q}}(1+\delta)^{k m} \lambda^{-(k m+m) / 2} k^{i_{1}+\cdots+i_{q}} .
\end{aligned}
$$

Using $(1+\delta) \leq \lambda^{1 / 24}$ we can bound this expression by

$$
\bar{K}_{m} \lambda^{-k(m / 2-1 / 24)} k^{m} \leq \bar{K}_{m} \lambda^{-k 5 / 4} k^{m}
$$

where $\bar{K}_{m}$ is obtainable out of the $\sigma$ 's, $K_{q}, c_{1} \cdots c_{m}$ and, hence, independent of $k$ and uniform in $r$ in a $C^{m}\left(B_{\zeta}\right)$ neighborhood.

If $q=2,1,0$ we can obtain similar bounds. Summing them all up we obtain

$$
\left\|\lambda^{k} D^{m} R \circ r^{-k}\right\|_{C^{0}\left(B_{\zeta}\right)} \leq \lambda^{k} \bar{K}_{m} \lambda^{-5 / 4} k^{m}
$$

which indeed can be summed uniformly in $k$. This finishes the proof of Lemma 5.1.

Now we prove Lemmas 5.2 and 5.3.

Lemma 5.3 is almost immediate if we recall that the Taylor series for $R$ at the origin begins with cubic terms.

Notice that the assumption of the lemma imply that

$$
\left\|r^{-1}(\underline{x}, \underline{y}, z)\right\| \leq \frac{(1+\delta)}{\sqrt{\lambda}}\|(\underline{x}, \underline{y}, z)\|
$$

for $(\underline{x}, \underline{y}, z) \in B_{\zeta}$.

The proof of Lemma 5.2 is slightly more involved and depends on the following counting argument.

Lemma 5.4. If $m \geq 1, D^{m} r^{-k}(\underline{x}, \underline{y}, z)$ is an expression involving $T(m, k)$ terms, each with at most $F(m, k)$ factors. Each factor is of the form

$$
\left(D^{l} r^{-1}\right) \circ r^{-j} \text { for } 1 \leq l \leq m, 0 \leq j \leq k-1 .
$$

Furthermore, in each term there are at least $(k-m)$ factors of the form $\operatorname{Dr}_{t}^{-1}\left(\underline{x}^{\prime}, \underline{y}^{\prime}, z^{\prime}\right)$ for some $\left(x^{\prime}, y^{\prime}, z\right) \in B_{r}$, and at most $m$ factors that are not of this form. Finally, $F(m, k) \leq k \cdot m$ and $T(m, k) \leq m ! k^{m}$.

Proof. For $m=1$,

$$
D\left(r^{-k}(\underline{x}, \underline{y}, z)\right)=\prod_{j=0}^{k-1}\left(D r^{-1}\right) \circ r^{-j}(\underline{x}, \underline{y}, z),
$$


and the conclusions are true for all $k$. We assume the lemma holds for $1 \leq$ $m \leq M$, and all $k$. Then

$$
D^{M+1}\left(r^{-k}(\underline{x}, \underline{y}, z)\right)=D\left(D^{M} r^{-k}(\underline{x}, \underline{y}, z)\right) .
$$

The last derivative will act on one of the $T(M, k)$ terms in the expression for $D^{M} r^{-k}$. In doing so it can (by the product rule) replace that one term by $F(M, k)$ others, each of which (by the chain rule) has at most $F(M, k)+$ $k$ factors. Thus, $T(M+1, k) \leq T(M, k) \cdot F(M, k)$ and $F(M+1, k) \leq$ $F(M, k)+k$. Since $F(1, k)=k$ and $T(1, k)=1$, the estimates on $T(m, k)$ and $F(m, k)$ follow.

The remaining claims in the lemma follow if we note that when we differentiate a factor like $\left(D^{l} r\right) \circ r^{-j}$, we generate factors of the same form, but with different values of $l$ and $j$. Also, by the chain rule, such a differentiation will increase the number of factors of the form $\mathrm{Dr}^{-1}$, and produce at most one more factor not of this form (unless when $j=0$, in which case it can reduce the number of factors of $D r_{t}^{-1}$, but by no more than one).

The proof of Lemma 5.2 now follows easily

$$
\left\|D^{m} r^{-k}\right\|_{C^{0}\left(B_{\xi}\right)} \leq m ! k^{m} B^{m} \frac{(1+\delta)^{k-m}}{(\lambda) \frac{k-m}{2}} .
$$

Here the factor of $m ! k^{m}$ bounds the number of terms in the expression for $D^{m} r^{-k}, B=\sup _{j=2, \ldots, m}\left\|D^{j} r^{-1}\right\|_{C^{0}\left(B_{\xi}\right)}$, so $B^{m}$ bounds the contribution of terms not of the form $D r^{-1}$. Finally, each factor of $D r^{-1}$ is bounded by $(1+\delta) / \sqrt{\lambda^{0}}$, by Lemma 5.3. Defining $C_{m}=m ! B^{m}(1+\delta)^{-m}$ yields Lemma 5.2 .

Remark. Analytic regularity of the solutions when the data are analytic is very easy to obtain because it suffices to check that $\sum \lambda^{k}\left\|R \circ r^{-k}\right\|$ is summable when the norm is the supremum of the functions in a complex extension of $B_{\zeta}$. This is the same computation to establish (5.10) when $m=0$.

Remark. The solution of (5.10) is not unique. One can readily construct other solutions by iterating relation $(5.10)$ backwards.

Nevertheless, the solution $G$ we constructed is unique among the functions that tend to zero faster than $\|x\|^{2+1 / 100}$.

In effect, if $G$ satisfies (5.10) then

$$
\begin{aligned}
G(x) & =R(x)+\lambda G\left(r^{-1}(x)\right) \\
& =R(x)+\lambda R\left(r^{-1}(x)\right)+\lambda^{2} G\left(r^{-2} x\right) \\
& \left.=\sum_{k=0}^{N} \lambda^{k} R\left(r^{-k}(x)\right)+\lambda^{N+1} G\left(r^{-N+1}\right)\right) .
\end{aligned}
$$

If $G(x)=O\left(\|x\|^{3-1 / 26}\right)$ the last term goes to zero using Lemma 5.3. 
Conversely, using the bounds in the first part of Lemma 5.3 one can easily check that $G(x)=O\left(\|x\|^{3-2 / 26}\right)$ since it is $C^{\infty}$, the two first derivatives should vanish, so that $G(x)=O\left(\|x\|^{3}\right)$.

The fact that $G_{t}(x)$ vanishes up to high order for all $t$ guarantees that the isotopy generated is tangent to the identity (as we will see, this result is also true for the full equation with variable $\lambda$ ).

Even if it is not necessary for the purpose of establishing the main theorem of this paper, we will now prove a strengthening of Lemma 5.1 which can be useful in other contexts.

Lemma 5.5. If $R_{t}, r_{t}$ are $C^{\infty}$ families of functions and diffeomorphisms satisfying the hypothesis of Lemma 5.1, the solutions constructed in this lemma, depend on the parameter in a $C^{\infty}$ fashion.

Proof of Lemma 5.5. Just as before, it suffices to show that

$$
\sum \lambda^{k}\left\|\left(\frac{\partial}{\partial t}\right)^{m} R_{t} \circ\left(r_{t}^{-k}\right)\right\|_{C^{0}\left(B_{\zeta}\right)}<\infty
$$

for all $m$.

The proof will follow along the same lines as before but it is slightly more delicate since the derivatives with respect to parameters propagate in a more complicated fashion than the chain rule.

In the following lemmas $C_{m}$ will denote constants independent of $k$, which can be chosen uniformly for a $C^{m}$ ball of $r_{t}$ 's $R_{t}$.

Unless otherwise specified all norms are in the sense of $C^{0}\left(B_{\zeta}\right)$ and the hypothesis of Lemma 5.5 will be assumed.

\section{Lemma 5.6.}

$$
\begin{aligned}
\left\|\left[\left(\frac{\partial}{\partial t}\right)^{m} R_{t}\right]\left(r_{t}^{-k}\right)\right\| & \leq C_{m}(1+\delta)^{k} \lambda^{-3 k / 2}, \\
\left\|\left[D\left(\frac{\partial}{\partial t}\right)^{m} R_{t}\right]\left(r_{t}^{-k}\right)\right\| & \leq C_{m}(1+\delta)^{k} \lambda^{-k}, \\
\left\|\left[D^{2}\left(\frac{\partial}{\partial t}\right)^{m} R_{t}\right]\left(r_{t}^{-k}\right)\right\| & \leq C_{m}(1+\delta)^{k} \lambda^{-k / 2}, \\
\left\|\left[D^{q}\left(\frac{\partial}{\partial t}\right)^{m} R_{t}\right]\left(r_{t}^{-k}\right)\right\| & \leq C_{m}, \quad q \geq 3 .
\end{aligned}
$$

The proof is very simple if we recall that $R_{t}(0)=0$ hence $(\partial / \partial t)^{m} R_{t}(0)=0$ and similarly for $D R_{t}, D^{2} R_{t}$ so that $R_{t}(x)$ is cubic in $x$.

Lemma 5.7. $\left\|\left[(\partial / \partial t)^{m} r_{t}^{-1}\right]\left(r_{t}^{-k}\right)\right\| \leq C_{m}(1+\delta)^{m} \lambda^{-k}$.

Proof of Lemma 5.7. $r_{t}^{-1}(0)=0$ and $D r_{t}^{-1}(0)=\varphi^{-1}$ so that $(\partial / \partial t)^{m} r_{t}^{-1}(0)=$ 0 and $D(\partial / \partial t)^{m} r_{t}^{-1}(0)=0$, and it suffices to take $C_{m}$ equal to a bound on the second derivative of $[\partial / \partial t]^{m} r_{t}^{-1}$. 
Lemma 5.8. $\left\|(\partial / \partial t)^{m} r_{t}^{-k}\right\| \leq C_{m}(k+1)^{2 m}(1+\delta)^{m} \lambda^{-k / 2}$.

Proof of Lemma 5.8. By applying the elementary rules of differentiation we can write down $(\partial / \partial t)^{m} r_{t}^{-k}$ as a sum of $T(m, k)$ terms each one of them containing not more than $F(m, k)$ factors of the form $\left(D^{i}(\partial / \partial t)^{j} r_{t}^{-1}\right)\left(r_{t}^{-l}\right)$ and $0 \leq l \leq k, 0 \leq i+j \leq m$.

The reasoning is pretty much as in the previous case. The main difference is that the number of terms grows much faster. In effect the derivative of an elementary factor with respect to $t$ is not just a product of factors but, rather, it contains $(l+1)$ terms, each one corresponding to one of the $t$ 's appearing.

So, when we take an extra derivative, the number of terms grows because of the application of the product rule but also because each one of the elementary factors gives rise to extra terms.

Hence, $T(m+1, k) \leq(k+1) F(m, k) T(m, k)$.

The maximum number of factors of a term grows as before:

$$
F(m+1, k) \leq(k+1)+F(m, k) \text {. }
$$

Hence we have that

$$
F(m, k) \leq m(k+1), \quad T(m, k) \leq(k+1)^{2 m} m ! .
$$

To finish the proof of the lemma it is convenient to add a counting device which we call "weight". The weight of a term is the weight of its elementary factors and the weight of elementary factors of the form $\left(D(\partial / \partial t)^{m} r_{t}^{-1}\right) \circ r_{t}^{-l}$ is 1 , the weight of $\left((\partial / \partial t)^{m} r_{t}^{-1}\right) \circ r_{t}^{-l}$ is $l$ and that of other elementary factors is zero.

We observe that, when we take the derivative of one term it gives rise to several terms all of which have greater or equal weight.

We also observe that the number of factors of weight zero in the terms of a derivative is at most one more than the numbers of factors of weight zero in the original term. Hence, the number of factors of weight zero in each of the terms of $(\partial / \partial t)^{n} r_{t}^{-k}$ is at most $m$.

Finally, observe that, if we call $B_{m}=\sup _{i, j \leq m}\left\|D^{i}(\partial / \partial t)^{j} r_{t}^{-1}\right\|_{c^{0}}$ then, we can bound all the factors of weight zero by $B_{m}$, also, we can bound all the factors of positive weight by $\left[(1+\delta) \lambda^{-1 / 2}\right]$ weight.

Since the weight of the first derivative is $k$ and it increases, it follows that each one of the terms in the $m$ th derivative can be bounded by $B_{m}^{m}\left[(s+\delta) \lambda^{-1 / 2}\right]^{k} \quad$ (using the bounds discussed before for each of the factors and the fact that a term does not have more than $m$ factors of weight zero).

The bounds of the lemma follow by multiplying the bounds for each term by the number of terms.

Proving Lemma 5.5 out of the lemmas we have established is now very simple. We just expand $(\partial / \partial t)^{m}\left(R_{t} \circ r_{t}^{-k}\right)$ without attempting to expand $(\partial / \partial t)^{l} r_{t}^{-k}$. Each one of the terms has an exponential bound but the reasoning is different for each one of the cases of Lemma 5.6. 
All that remains to complete the proof of our Main Lemma is to show how we can reduce equation (5.8) to (5.9).

We begin with

$$
R_{t}-G_{t}+\left(\lambda_{t}^{(r)} G_{t}\right) \circ r_{t}^{-1}=0
$$

Define a function $\psi_{t}$ implicitly by

$$
\lambda_{t}^{(r)} \circ r_{t}^{-1}=\lambda^{0} \frac{\psi_{t} \circ r_{t}^{-1}}{\psi_{t}}
$$

We show below that $\psi_{t}$ is well defined, nonzero on some neighborhood of the origin, and smooth. Inserting (5.13) into (5.8) gives

$$
R_{t}-G_{t}+\lambda^{0}\left\{\frac{\left(\psi_{t} G_{t}\right) \circ r_{t}^{-1}}{\psi_{t}}\right\}=0
$$

or

$$
\left(\psi_{t} R_{t}\right)-\left(\psi_{t} G_{t}\right)+\lambda^{0}\left(\psi_{t} G_{t}\right) \circ r_{t}^{-1}=0 .
$$

Defining $\psi_{t} R_{t}=\widetilde{R}_{t}$ and $\psi_{t} G_{t}=\widetilde{G}_{t}$, this is equivalent to (5.9). Thus, we are done if we can construct $\psi_{t}$ with the stated properties. First divide (5.13) by $\lambda^{0}$, and then take logarithms of both sides of the equation to obtain

$$
\log \left[\psi_{t} \circ r_{t}^{-1}\right]-\log \psi_{t}=\log \left[\frac{\lambda_{t}^{(r)} \circ r_{t}^{-1}}{\lambda^{0}}\right] \text {. }
$$

If we define

$$
V_{t}=\log \left[\frac{\lambda_{t}^{(r)} \circ r_{t}^{-1}}{\lambda^{0}}\right] \text { (which is defined at least on }
$$

some neighborhood of the identity)

and $Y_{t}=\log \psi_{t}(5.14)$ becomes

$$
V_{t}+Y_{t}-Y_{t} \circ r_{t}^{-1}=0
$$

another cohomology equation but simpler since there is no extra factor $\lambda$ ! If we recall that $r_{t}$ is tangent to $\varphi$ at the origin we readily find that $\lambda_{t}^{(r)}(\underline{0}, \underline{0}, 0)=\lambda^{0}$. Thus the Taylor series for $V_{t}$ at the origin begins with linear terms or higher. From a very similar discussion to the one establishing Lemma 5.1, we know that

$$
Y_{t}=\sum_{k=0}^{\infty} V_{t} \circ r_{t}^{-k}
$$

will solve (5.15) if we can show that the right-hand side converges. But then, exponentiating $Y_{t}$ we get a nonzero $\psi_{t}$ as desired.

The proof of convergence and smoothness of $Y_{t}$ is similar but much simpler than the proof of Lemma 5.1. 
Lemma 5.2 is unchanged and the only thing we need to show convergence is the Faa-di Bruno formula and uniform bounds for the derivatives of $V$.

Since all these bounds are uniform in a $C^{m}$ ball, we automatically get continuous dependence on parameters.

We get, as claimed, a continuous family of $C^{\infty}$ functions solving the cohomology equation and each one of the functions is zero up to cubic order.

This proves the Main Lemma.

We just remark that it is possible to get $C^{\infty}$ dependence on parameters for the second-easy-cohomology equation so that it is possible to get smooth dependence on parameters for the full cohomology equation equivalent to conjugation.

Remark. The equation we were aiming to solve in the Main Lemma is a conjugacy equation and it would have been very natural to study it using Zehnder's theorem [Z].

One advantage of such an approach is that it only requires solvability of the cohomology equation at one diffeomorphism. Our method requires solvability in a neighborhood and continuous dependence of the solution on parameters.

The method presented here has the advantage that we do not need that the estimates be "tame".

In the problem considered here, both methods would have been applicable but, in [BLW] we consider a cohomology equation with solutions that do not satisfy tame estimates. In contrast, for the small divisor problems considered in [Z] our method is not quite applicable but Zehnder's works perfectly.

One slight complication in the application of Zehnder's method to the problems considered here is that the expansiveness of the map causes a mismatch of domains and the iterative procedure involves also choices of domains. Coping with such a problem-it is done e.g. in [Z2]-is well understood.

\section{APPENDIX A}

Theorem. The abelianisation $H_{1}\left(\operatorname{Diff}_{+}^{\infty}\left(\mathbf{R}^{2 n+1}, \omega, 0\right)_{*}\right)$ of the group

$$
\operatorname{Diff}_{+}^{\infty}\left(\mathbf{R}^{2 n+1}, \omega, 0\right)_{*}
$$

is isomorphic to the multiplicative group $\mathbf{R}^{+}$.

Proof. Denote by $G$ the group $\operatorname{Diff}_{+}^{\infty}\left(\mathbf{R}^{2 n+1}, \omega, 0\right)_{*}$ and by $G_{*}$ its subgroup formed by those elements which are tangent to the identity at the origin. According to $\S 2$, it is enough to show that $G_{*}=\left[G, G_{*}\right]$.

Let $\psi \in G_{*}$; we may assume $\psi$ is as close to the identity as we wish, without loss of generality. (Indeed let $\psi_{t} \in G_{*}$ be an isotopy from $\psi$ to the identity in $G_{*}$, then $\psi=\psi_{0} \circ \cdots \circ \psi_{N-1}$ with $\psi_{i}=\psi_{(N-i) / N} \cdot \psi_{(N-(i+1)) / N}^{-1}$ which can be made as small as we want by taking $N$ large enough.)

The main result of this paper asserts that on a sufficiently small neighborhood $U^{\prime}$ of the origin there is a contact diffeomorphism $u$ fixing 0 and tangent to the identity there, such that $\psi=\left[\varphi_{\lambda}, u\right]$ on a neighborhood $U \subset U^{\prime}$ of the 
origin. Here $\varphi_{\lambda}$ is some contact homothety and $u$ may be assumed to have a compact support in $U^{\prime}$. The diffeomorphism $\sigma=\psi \cdot\left[\varphi_{\lambda}, u\right]^{-1}$ is supported outside the neighborhood $U$ of the origin.

A verbatim repetition of the arguments of [BP and $\mathrm{P}$ ], which are the contact version of those of McDuff [MD], show that $\sigma$ can be written as a product of 2 commutators: $\sigma=\left[\alpha_{1}, \beta_{1}\right] \cdot\left[\alpha_{2}, \beta_{2}\right]$ where $\beta_{i} \in G_{*}$ are supported outside a small ball about the origin and $\alpha_{i} \in G$ are conjugate in $G$ to some contact homothety. (In fact, that $\sigma$ is supported away from the origin even facilitates the argument at p. 47 in [BP], where in our case, $\mu_{0}$ will be simply a contact homothety.)

Hence $\sigma \in\left[G, G_{*}\right]$ and therefore $\psi \in\left[G, G_{*}\right]$.

Acknowledgments. It is a pleasure to thank S. Hurder and L. Vaserstein for useful discussions, and J.-P. Eckmann for a careful reading of the manuscript.

\section{REFERENCES}

[AR] R. Abraham and J. Robbin, Transversal mappings and flows, Benjamin, New York, 1967.

[BP] A. Banyaga and J. Pulido, On the group of contact diffeomorphisms of $\mathbf{R}^{2 n+1}$, Bol. Soc. Mat. Mexicana 23 (1978), 43-47.

[BLW] A. Banyaga, R. de La Llave and E. Wayne, Cohomology equations near hyperbolic points, and applications to normal forms of symplectic systems, in preparation.

[C] M. Chaperon, Géométrie différentielle et singularités de systèmes dynamiques, Astérisque 138139 (1986).

[D] L. E. Dickson, Theory of linear groups in arbitrary field, Trans. Amer. Math. Soc. 2 (1901), 363-394.

[G] J. Gray, Some global properties of contact structures, Ann. of Math. 69 (1959), 421-450.

[HS] P. Hilton and U. Stammback, Homological algebra, Graduate Texts in Math., no. 4, SpringerVerlag, Berlin, 1970.

[I] M. C. Irwin, On the smoothness of the composition map, Quart. J. Math. Oxford 2 (1972), 113-133.

[L] H. B. Lawson, The qualitative theory of foliations, CBMS Regional Conf. Ser. in Math., no. 27, Amer. Math. Soc., Providence, R.I.

[Li] P. Liberman, Sur les automorphismes infinitesimaux des structures symplectiques et des structures de Contact, Colloque de Géométrie Différentelle Globale Bruxelles (1958), Louvain, 1959.

[LMM] R. de la Llave, J. M. Marco and R. Moriyón, Canonical perturbation theory of Anosov systems and regularity results for the Livsic cohomology equation, Ann. of Math. 123 (1986), 537-611.

[Ly] V. V. Lychagin, On sufficient orbits of a group of contact diffeomorphisms, Math. USSR-Sb. 33 (1977), 223-242.

[Ly2] __, Local classification of non linear first order partial differential equations, Russian Math. Surveys 30 (1975), 105-175.

[MA] J. N. Mather, On the homology of Haefliger's classifying space, Differential Topology (V. Villani, ed.), C.I.M.E., V.III, Ciclo 1976, Varena, Italy.

[MD] D. McDuff, On the group of volume preserving diffeomorphisms of $\mathbf{R}^{n}$, Trans. Amer. Math. Soc. 261 (1980), 103-113.

[Ne] E. Nelson, Topics in dynamics. I: Flows, Princeton Univ. Press, Princeton, N.J., 1969. 
[P] J. Pulido, An equation of contact vector fields and the group of contact diffeomorphisms, Thesis, Princeton Univ., 1981.

[R] J. Robbin, On the existence theorem for differential equations, Proc. Amer. Math. Soc. 19 (1968), 1005-1006.

[S] F. Sergeraert, Feuilletages et difféomorphismes infiniment tangents a l'identité, Invent. Math. 39 (1977), 253-275.

[Se] G. Sell, Smooth linearization near a fixed point, Amer. J. Math. 107 (1985), 1035-1091.

[St] S. Sternberg, On the structure of local homeomorphisms of Euclidean n-space. II, Amer. J. Math. 80 (1958), 623-631.

[St2] __, The structure of local homeomorphisms. III, Amer. J. Math. 81 (1959), 578-604.

[T] F. Takens, Normal forms for certain singularities of vector fields, Ann. Inst. Fourier (Grenoble) 23 (1973), 163-195.

[Z] E. Zehnder, Generalized implicit functions theorems with applications to some small divisor problems. I, Comm. Pure Appl. Math. 28 (1975), 91-140; II, Comm. Pure Appl. Math. 29 (1976), 41-111.

[Z2] _ A simple proof of a generalization of a theorem by C. L. Siegel in geometry and topology (Palis et al., ed.), Lecture Notes in Math., vol. 597, Springer-Verlag, New York, 1977.

Department of Mathematics, Pennsylvania State University, University Park, PennSYLVANIA 16802 (Current address of Augustin Banyaga and C. Eugene Wayne)

Department of Mathematics, Princeton University, Princeton, New Jersey 08544 (Current address of Rafael de la Llave) 